\title{
Revenances de la Corriveau
}

\section{Sophie Ménard}

\section{OpenEdition}

Journals

Édition électronique

URL : https://journals.openedition.org/clo/6157

DOI : $10.4000 /$ clo.6157

ISSN : 2266-1816

\section{Éditeur}

INALCO

\section{Édition imprimée}

Date de publication : 18 juin 2019

Pagination : 215-222

ISBN : 978-2-85831-348-8

ISSN : 0396-891X

\section{Référence électronique}

Sophie Ménard, « Revenances de la Corriveau », Cahiers de littérature orale [En ligne], 85 | 2019, mis en ligne le 19 juin 2020, consulté le 28 juin 2022. URL : http://journals.openedition.org/clo/6157 ; DOI : https://doi.org/10.4000/clo.6157

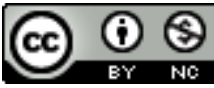

Cahiers de littérature orale est mis à disposition selon les termes de la Licence Creative Commons Attribution - Pas d'Utilisation Commerciale 4.0 International. 


\title{
Revenances de la Corriveau
}

\author{
Sophie MÉnard \\ Université de Montréal
}

Je ne me rappelle pas la première fois où j'ai entendu parler de la Corriveau. Cela doit remonter à loin, mais j'ai l'impression que cette figure a toujours été là dans mon histoire, en tout cas, dans l'histoire du Québec. La Corriveau, c'était un récit de sorcière à faire peur qu'on (se) racontait. Une femme avait un jour, il y a très longtemps, au temps de la défaite française, assassiné ses maris (combien y en a-t-il déjà ? un, deux ou sept ?). Pour ce crime, on l'avait, dans mon imaginaire, enfermée vivante dans une cage suspendue au-dessus d'un carrefour, qui menait à la ville de Lévis, où elle hante depuis les passants. Encore aujourd'hui, si on y prête bien attention, dans certaines rues, on peut entendre ses cris et lamentations maléfiques.

Cette légende ici déformée par le passage du temps et des voix et par mes lectures récentes a une origine historique que retrace avec minutie l'ouvrage de référence enfin publié de Luc Lacourcière, ethnographe, folkloriste et écrivain québécois : La Corriveau. La formation d'une légende ${ }^{1}$. Condamnée à mort par une cour martiale anglaise, en 1763, pendue et ensuite suspendue publiquement, pendant quarante jours, dans une cage de fer, sous forme d'exosquelette, pour le meurtre de son second mari, Marie-Josephte Corriveau est, selon Lacourcière, la femme qui, dans toute l'histoire canadienne, a la plus mauvaise réputation ${ }^{2}$. C'est certainement pourquoi, 250 ans après le drame, elle continue de me fasciner et de troubler l'imaginaire québécois.

1. LACOURCIÈRE, 2017.

2.Ibid., p. 39. 
À la recherche des signes, imprimés et oraux, illustrant la « formation d'une légende » québécoise, ce livre posthume ${ }^{3}$, paru dans l'incontournable collection «Les Archives de folklore » que Lacourcière a fondée en 1946, rassemble les trois principaux articles du fondateur du premier programme d'enseignement universitaire de l'ethnologie au Canada, dans lesquels il démêle l'écheveau du destin « de cette passionnante énigme ${ }^{4}$ ». On rappellera que Lacourcière, formé à la folkloristique auprès de Marius Barbeau, a publié des études sur la tradition orale et sur la chanson traditionnelle (notamment Essais sur Émile Nelligan et sur la chanson populaire). Outre la mise en chantier du Catalogue raisonné du Conte populaire en Amérique du Nord et de La Bibliographie raisonnée du folklore français d'Amérique (qui ne sont pas publiés), il s'intéresse, vers la fin des années 1950, à la Corriveau, multipliant les conférences (plus d'une quinzaine) sur le sujet. C'est cette documentation qu'a rassemblée l'ethnologue et spécialiste du patrimoine oral et de l'histoire régionale du Saguenay-Lac-Saint-Jean, Bertrand Bergeron, et que publie Jean-Pierre Pichette dans la collection, toujours active, des « Archives du folklore $\gg$.

Ce dossier captivant sur la Corriveau, considéré par Bertrand Bergeron comme un « classique de l'ethnologie québécoise ${ }^{5} »$, est serti d'un triple discours. Le premier traite le récit du crime de la Corriveau dans une perspective historique, à partir principalement des sources judiciaires. Le deuxième nous le présente, sous l'angle littéraire, comme une reconfiguration scripturale. Le dernier nous le livre du riche point de vue de la culture orale et du processus de « légendarisation ».

\section{Le destin criminel : le fait divers}

1763, la date est fatidique dans l'histoire du Canada. Le destin de ce jeune pays est scellé depuis la fin de la Guerre de la Conquête, et le traité de Paris, ratifié en février 1763, sanctionne la cession de la Nouvelle-France à la couronne britannique. Le crime commis pendant la nuit du 26 au 27 janvier a lieu dans un moment historique liminaire ; c'est que, depuis trois ans déjà, le Canada vit sous la loi martiale : « le drame qui s'est déroulé à Saint-Vallier impliquait des vaincus qui allaient subir la justice militaire des vainqueurs dans la langue de ces derniers. Un drame domestique donc se déroulant à l'intérieur d'un drame national en

3. Luc Lacourcière, né en 1901, est décédé en 1989.

4. LACOURCIÈRE, 2017, p. 40.

5.Ibid., p. 35. 
quelque sorte ${ }^{6} \gg$, écrit Bertrand Bergeron dans son excellente introduction qui relate les temps forts de la légende et, surtout, qui redonne au travail minutieux de Lacourcière la place fondatrice lui revenant.

Dans cette première partie, Lacourcière, s'appuyant sur une riche documentation historique, reconstitue le fait divers et le double procès du crime. La première procédure concerne Joseph Corriveau, le père de « la » Corriveau, accusé d'avoir tué son gendre et voisin, Louis-Étienne Dodier, avec qui - tout le village le sait - il a eu de nombreuses disputes. Sont également accusées sa complice, Marie-Josephte Corriveau, veuve du dit Dodier, et une servante, Isabelle Sylvain. À l'issue du procès, Joseph est condamné à la pendaison, sa fille au marquage et au fouet, la domestique à trente coups de fouet et à la flétrissure des parjures. Or, le père Corriveau finit par avouer son innocence et par dénoncer sa fille pour éviter de mettre en péril son âme. Confrontée par les rétractations de son père, Marie-Josephte confesse son crime et est alors vouée à la pendaison dans les chaînes. C'est cette cage (utilisée uniquement à deux reprises dans l'histoire du Canada) qui, on s'en doute, marquera durablement les imaginaires.

Mais qu'est-ce qui explique le geste ? Peu nous est dévoilé sur le sujet. Est-il né d'une impulsion ? D’une rage démente ? D’une préméditation ? Seule nous est donnée la déclaration officielle de Marie-Josephte déclarant avoir assassiné son mari « pendant la nuit alors qu'il dormait dans son lit » : « elle l'a fait avec une petite hache » et « elle n'a été incitée ni aidée par aucune autre personne à le faire [...]. Elle ajoute que c'est vraiment dû en grande partie aux mauvais traitements de son mari si elle est coupable de ce crime ${ }^{7} \gg$. La mésentente des époux Corriveau est de notoriété publique; des témoins, notamment les voisins, témoigneront en ce sens, comme ce Claude Dion, qui rapporte quelques paroles prononcées par la Corriveau à son mari, la veille du crime : «Tu manges bien, mais ne travailles pas beaucoup ; peut-être que tu ne mangeras pas très longtemps ${ }^{8} ! \gg$ Ce voisin affirme de surcroît que la femme lui faisait la cour et qu'elle souhaitait se débarrasser de son mari.

On regrette, à ce titre, de ne pas entendre la Corriveau qui n'a pas droit de parole dans cette histoire dont elle est pourtant l'héroïne. Le livre de Lacourcière, très exhaustif sur le plan de l'archive historique, reste silencieux sur la vie quotidienne de cette femme. On aurait aimé obtenir la reproduction ou la retranscription complète du dossier des témoins qui, peut-être, aurait pu
6.Ibid., p. 19.
7.Ibid., p. 56.
8.Ibid., p. 52. 
nous parler des extravagances, des bizarreries, des afflictions, de l'ivrognerie de la Corriveau. Qui était-elle de son vivant ? Pourquoi a-t-elle choisi d'épouser en secondes noces ce Dodier ? S'agissait-il d'une mésalliance (elle est veuve et mère de trois enfants) et d'un mariage en gendre (le père de la Corriveau réside dans la maison voisine, les époux partagent avec lui le four à pain et la mésentente à ce sujet aurait mené le père et le gendre aux coups) ? On sait que la discorde familiale est connue : pourquoi dès lors le village n'a-t-il pas réglé la question par les voies et la justice coutumières ? Un charivari aurait-il pu éviter le pire ? Il manque ici une véritable enquête d'ethnographie historique, à la manière de Ginzburg ou de Le Roy Ladurie, qui ressusciterait la réalité de Saint-Vallier dans les années précédant le crime : démographie, environnement, métiers principaux, étude socio-politique, vie matérielle, croyances, mentalités, ritualité, etc. Une telle entreprise aurait-elle permis de mieux saisir la dynamique villageoise dans laquelle vivait Marie-Josephte, de mieux cerner la trajectoire de cette vie qui ne commence pas en 1763 ? Sans doute, considérant la minutie du travail de Lacourcière dans les archives, faut-il croire que ce sont les documents d'ethnologie paysanne qui font défaut. Dès lors, le mystère restera entier...

\section{Le destin littéraire}

La littérature permet de suppléer aux manques de documents factographiques. Philippe Aubert de Gaspé (père), « le mythographe par excellence de la Corriveau $^{9} \gg$, Louis Fréchette et William Kirby contribuent à donner ses lettres de noblesse au crime de la Corriveau et à l'inscrire dans les annales de la jeune culture de l'imprimé et de la littérature du Canada. Le récit princeps des Anciens Canadiens (1863) met en scène la rencontre entre François Dubé et la Corriveau, qui apparaît sous les traits de la figure du cauchemar. Voulant traverser, sur le dos du chrétien, le fleuve Saint-Laurent, sacré et interdit, afin de participer à la danse diabolique de l'île d'Orléans, la Corriveau pèse, avec tout le poids de sa cage, sur le dormeur pour l'obliger à lui rendre le service. Ce texte continue d'influencer plusieurs des versions orales recueillies au cours $\mathrm{du} \mathrm{Xx}^{\mathrm{e}}$ siècle par les ethnologues québécois. «Une relique », le conte fantastique de Fréchette, publié en 1898, ajoute à l'histoire le crime du premier mari, assassiné au moyen du plomb fondu versé dans les oreilles, alors qu'avant lui Kirby, dans The Golden Dog (1877), fait de la criminelle une vieille sorcière et meurtrière à gages.

9.Ibid., p. 79 . 
Outre ces trois références majeures, Lacourcière recense minutieusement avant l'invention d'internet et des précieux moteurs de recherche numérique toutes les apparitions imprimées de la Corriveau depuis le procès jusqu'aux années 1970, soulignant au passage les erreurs qui « donne[nt] à des motifs apocryphes le crédit de l'imprimé auprès des lecteurs non avertis ${ }^{10} \gg$. La confrontation avec les sources permet d'étudier les variations et de mesurer les écarts différentiels entre les versions. C'est la présence des formes folkloriques de la culture, appropriée et retravaillée par le texte littéraire, tout autant que les constructions spécifiques de la culture imprimée qui l'intéresse.

La publication posthume en volume des articles de Lacourcière, publiés successivement en 1968, 1969 et 1973, fait d'ailleurs suite à la parution d'un ouvrage présentant la biographique et la légende de la célèbre meurtrière de Saint-Vallier : La Corriveau. De l'Histoire à la légende de Catherine Ferland et Dave Corriveau ${ }^{11}$. Ce livre complète, entre autres, l'inventaire lacourciérien des apparitions de la Corriveau dans le monde contemporain (des années 1970 au début des années 2000), où la figure change, actualisée par les discours féministes et nationalistes. Citons ici pour le plaisir de les lire et pour inviter le lecteur à aller les écouter les trois premiers couplets (sur sept) de l'excellente chanson « La corrida de la Corriveau » du groupe québécois Mes Aieux :

Uno !

Le premier mari de la Corriveau

Était un bonhomme qui dormait sûrement un peu trop

Car, une nuit de sommeil trop agité,

Il s'est étouffé à mort avec son oreiller

Dos!

La veuve s'est remariée, ce ne fut pas trop long

Avec un alcoolique qui faisait des dépressions

On l'a r'trouvé pendu d'une drôle de façon

La corde attachée à la selle de son étalon

La la la...

Tres!

« Jamais deux sans trois » comme le dit le dicton

Sur un fondeur de cuillères, elle jeta son dévolu

10. Ibid., p. 85.

11. Ferland \& Corriveau, 2014. 
Mais le pauvre étant victime d'une distraction

Dans l'oreille s'est versé de l'étain fondu (hayayaye)

$[\ldots]^{12}$.

La légende est bien vivante. Cette chanson, on s'en doute, n'aurait certainement pas déplu au grand chercheur de l'Université Laval, lui qui a également recensé les multiples présences de la Corriveau dans des médias variés : du ballet dramatique sur chansons originales de Gilles Vigneault à une affiche placardée en 1970 dans le pavillon des légendes canadiennes de la Terre des hommes en passant par les céramiques, dessins, peintures, etc., la figure de la Corriveau est cernée, poursuivie, dénichée dans ses moindres apparitions.

\section{Le destin légendaire}

« [E]xemple saisissant des transformations des faits divers dans la mémoire collective $^{13} \gg$, les riches incarnations orales de la Corriveau confirment que cette Barbe bleue au féminin polarise « l'anathème du jugement populaire ${ }^{14}$ ». Elles l'associent, à cause de son supplice infernal, au surnaturel maléfique. La pendaison spectaculaire dans la cage motive en effet la formation d'un imaginaire collectif. D'ailleurs, la découverte récente de la cage au Peabody Essex Museum de Salem redonne à l'affaire un regain de popularité.

Trois motifs importants forment la légende. Le premier est l'héroïne principale : la Corriveau, que la tradition nomme toujours ainsi, est « une femme de mauvaise vie, une méchante femme, une sorcière qui parlait au diable, qui dansait avec le diable, une femme vendue au diable ${ }^{15} \gg$. Le second est le crime, plus souvent les crimes, et les moyens divers par lesquels elle s'y est prise pour réaliser ses forfaits. D'un à sept maris tués, parfois même des enfants, la Corriveau emploie plusieurs outils pour se débarrasser de ses époux encombrants : le plomb versé dans l'oreille, le poison, le broc à fumier ou le croc, l'étranglement, l'aiguille plantée dans le cœur, l'épingle au cerveau, le rasoir pour égorger. Certaines de ces techniques recoupent des motifs légendaires connus et relevés par Stith Thompson (S 111, S 112.3, S 113, S 115.2). Enfin, dernier noyau central de

12. Mes AḮux, 2001.

13. LACOURCIÈRE, 2017, p. 39.

14.Ibid., p. 70.

15.Ibid., p. 125. 
la légende : la cage de fer dont l'emplacement varie selon les narrateurs. Suivre les transformations, variations, reconversions des motifs de cette légende s'avère une aventure passionnante montrant que chaque époque a ses codes de sensibilités et de moralités à l'égard de cette femme (l'exemple de la censure en 1955 d'une cage en glace prévue au Carnaval de Québec est éclairant).

On ne peut que souligner le mérite de cette édition savante qui fera date. À titre d'ouvrage de référence sur la Corriveau, on aurait toutefois aimé une plus grande générosité dans la publication des archives de Lacourcière. Les documents reproduits en fac-similés demeurent souvent illisibles. L'absence de bibliographie est une lacune méthodologique importante : elle aurait assuré une meilleure chronologie de cette culture imprimée (plus de 75 auteurs différents sont archivés) et serait devenue un outil de recherche facilitant les travaux à venir. Une seule légende collectée (certes puissante et captivante) est donnée sur les cinquante-deux témoignages inédits consignés aux Archives de folklore et colligés entre les années 1952 et 1973 . Toutefois, une annexe contient une vingtaine de versions recueillies entre 1976 et 1980 par les élèves de secondaire II et III de Jean-Pierre Pichette (le directeur de la collection « Archives de folklore » et initiateur de ce livre posthume). Ce projet et cette publication de ce fonds personnel sont pour le moins fascinants : des jeunes de 13-14 ans, transformés en enquêteurs et folkloristes, ont collecté et retranscrit les légendes de leur environnement, de leur époque. Ce fonds nous incite à poursuivre le travail entamé par Pichette et Lacourcière et à penser une didactique du français qui prendrait davantage en compte la littérature orale ; magnifique matière, comme cette histoire de la Corriveau, à entendre, à mémoriser, à recueillir, mais aussi à enseigner pour apprendre à créer, à réciter, à lire et à écrire.

\section{Bibliographie}

Aubert de Gaspé Philippe, 1988 [1863], « La Corriveau » in Les Anciens Canadiens, Bibliothèque québécoise (Coll. Littérature), p. 65-76.

Du Berger Jean, 1989, « Un grand maître de la tradition. L'ethnologue Luc Lacourcière » in Cap-aux-Diamants. La revue d'histoire du Québec, vol. $4, n^{\circ} 4$, p. 19-22.

Ferland Catherine \& Corriveau Dave, 2014, La Corriveau. De l'Histoire à la légende, édition révisé, Septentrion, Québec, 392 p. 
FrÉCHETTE Louis, 2015 [1885], « Une relique (la cage de la Corriveau) » in Contes de Louis Fréchette, sous la direction d'Aurélien Boivin, Planète rebelle, Montréal, p. 90-103.

Kirby William, 2012 [1877], Le Chien d'or/The Golden Dog. A Legend of Quebec, édition par Mary Jane Edwards, McGill-Queen's University Press, Montréal/ Kingston, $1152 \mathrm{p}$.

LaCOURCière Luc, 2009 [1947], Essais sur Émile Nelligan et sur la chanson populaire, édition préparée par André Gervais, Fides, Québec, 450 p.

Lacourcière Luc, 2017, La Corriveau. La formation d'une légende, édition préparée par Bertrand Bergeron et Jean-Pierre Pichette, Presses de l'Université Laval, (Coll. Les Archives du folklore, $n^{\circ} 32$ ), Laval, 193 p.

Mes Aïeux, 2001, « La Corrida de la Corriveau » in Album Entre les branches, Disques Victoire, en ligne : https://www.youtube.com/ watch? $\mathrm{v}=\mathrm{zdOldNz} 4 \mathrm{uBY}$

Thompson Stith, 1955-1958, Motif-index of Folk-literature, édition révisée, Indiana University Press, Bloomington, en ligne : http://www.ruthenia.ru/ folklore/thompson/index.htm 